\title{
Strong \& Weak Lensing United: the Cluster Mass Distribution of RX J1347-1145
}

\author{
M. Bradač ${ }^{1,2}$, P. Schneider ${ }^{1}$, T. Erben ${ }^{1}$, and M. Lombardi ${ }^{1,3}$ \\ ${ }^{1}$ IAEF, University of Bonn, Auf dem Hügel 71, D-53121 Bonn, Germany \\ ${ }^{2}$ Max-Planck-Institut für Radioastronomie, Auf dem Hügel 69, D-53121 Bonn, Germany \\ ${ }^{3}$ ESO, Karl-Schwarzschild-Str. 2, D-85748 Garching bei München, Germany
}

\begin{abstract}
Weak gravitational lensing is considered to be one of the most powerful tools to study the mass and the mass distribution of galaxy clusters. However, the mass-sheet degeneracy transformation has limited its success. We present a novel method for a cluster mass reconstruction, which combines weak and strong lensing information on common scales and can as a consequence break the mass-sheet degeneracy. We extend the weak lensing formalism to the inner parts of the cluster, use redshift information of background sources and combine these with the constraints from multiple image systems. We apply the method to N-body simulations as well as to strong and weak lensing ground-based multi-colour data of RX J1347-1145, the most $\mathrm{X}$-ray luminous cluster known to date. If the redshift measurements of background sources (for strong and weak lensing) and the identification of the multiple-image system are correct, we estimate the enclosed cluster mass within $360 \mathrm{~h}^{-1} \mathrm{kpc}$ to $M\left(<360 \mathrm{~h}^{-1} \mathrm{kpc}\right)=(1.2 \pm 0.3) \times 10^{15} M_{\odot}$. With higher resolution (e.g. HST) imaging data, reliable multiple imaging information could be obtained and the reconstruction further improved.
\end{abstract}

\section{Introduction}

Clusters of galaxies have long been recognised as excellent laboratories for many cosmological studies. In particular, reliable mass-estimates are important since they provide constraints on the structure formation paradigm.

We use a combined strong and weak lensing mass reconstruction to determine the mass and the mass distribution of clusters. We reconstruct the gravitational potential $\psi$, since it locally determines both the lensing distortion (for weak lensing) as well as deflection (for strong lensing). The method extends the idea from Bartelmann et al. (1996); Seitz et al. (1998). Its novel feature is that we extend the weak lensing treatment to the critical parts of the cluster (i.e. $\kappa \gtrsim 1$ ) and directly include strong lensing information. In addition we use redshift information of the individual background sources and the source(s) being multiply imaged. This allows us to break the mass-sheet degeneracy and accurately measure the cluster mass and mass-distribution. The method is tested using simulations and applied to observational weak and strong lensing data of the cluster RX J1347-1145. In these proceedings we only briefly describe the method and the results, the full work will be published elsewhere (Bradač et al. 2004a,b).

\section{The cluster mass reconstruction method}

The main idea behind the method is to parametrise the cluster mass-distribution by a set of model parameters, where this parametrisation is chosen as generic as possible. The most straightforward way is to use the gravitational potential $\psi$ on a regular grid. We then define a penalty function $\chi^{2}$ and minimise it with respect to the values of $\psi_{k}$. 
The convergence $\kappa$, shear $\gamma$ and the deflection angle $\vec{\alpha}$ at an arbitrary position in the field are obtained by finite differencing and bilinear interpolation methods. The number of grid points we use for $\psi_{k}$ is $\left(N_{\mathrm{x}}+2\right) \times\left(N_{\mathrm{y}}+2\right)$; the extention by one row and column at each side is needed to be able to perform the finite differencing at each inner $N_{\mathrm{x}} \times N_{\mathrm{y}}$ grid point.

We define the $\chi^{2}$-function as follows

$$
\chi^{2}\left(\psi_{k}\right)=\chi_{\epsilon}^{2}\left(\psi_{k}\right)+\chi_{\mathrm{M}}^{2}\left(\psi_{k}\right)+\eta R\left(\psi_{k}\right) .
$$

$\chi_{\epsilon}^{2}\left(\psi_{k}\right)$ contains information from statistical weak lensing, whereas in $\chi_{\mathrm{M}}^{2}\left(\psi_{k}\right)$ we include the multiple imaging properties. $R\left(\psi_{k}\right)$ is a regularisation term multiplied by the regularisation parameter $\eta$. The regularisation is a linear function of the potential and disfavours any small-scale fluctuations in the potential.

In order to find the minimum $\chi^{2}$ solution, we consider

$$
\frac{\partial \chi^{2}\left(\psi_{k}\right)}{\partial \psi_{k}}=0 \text {. }
$$

This is in general a non-linear set of equations, we solve it in an iterative manner. We linearise this system and starting from some trial solution we repeat the procedure until convergence. Inverting the resulting matrix of $\sim N_{\mathrm{dim}}^{2}$ elements for finding a solution of linear system is difficult in general even for grids with small number of grid cells. However, as it turns out, the resulting matrix is sparse and it is therefore computationally inexpensive to solve the system.

Note, however, that the dimensionality of the problem is not $\left(N_{\mathrm{x}}+2\right) \times\left(N_{\mathrm{y}}+2\right)$. Because the transformation $\psi(\vec{\theta}) \rightarrow \psi(\vec{\theta})+\psi_{0}+\vec{a} \cdot \vec{\theta}$ leaves $\kappa$ and $\gamma$ invariant, and therefore the potential needs to be fixed at three points (see Seitz et al. 1998; Bartelmann et al. 1996). In addition, the transformation $\psi(\vec{\theta}) \rightarrow \psi(\vec{\theta})+\vec{a} \cdot \vec{\theta}$ changes the deflection angle $\vec{\alpha}$, however it only causes a translation of the source plane, which is not an observable. Therefore, even in the presence of strong lensing, three points of the potential need to be held fixed. Our ultimate aim is to make a reliable estimate for the cluster mass, therefore the mass-sheet degeneracy needs to be lifted. Hence in contrast to Seitz et al. (1998) the potential $\psi_{k}$ is not held fixed at an additional fourth point. The dimensionality of the problem is thus $N_{\text {dim }}=\left(N_{\mathrm{x}}+2\right)\left(N_{\mathrm{y}}+2\right)-3$.

Since the minimisation of $\chi_{\epsilon}^{2}$ can lead to a potential that reconstructs the noise in the data, the solution needs to be regularised. Even without the measurement errors, the intrinsic ellipticities would still produce pronounced small-scale peaks in the final reconstruction. Motivated by the success of a moving prior in maximum-entropy distribution (Seitz et al. 1998), we choose a very simple prescription for the regularisation function. We start off by a relatively coarse grid, since if $N_{\text {dim }}$ is much smaller than the number of galaxies, the resulting reconstruction will not be able to follow the noise pattern. We then gradually increase the number of grid points and compare the resulting surface mass density map $\kappa^{(n)}$ with that from the previous iteration $\kappa^{(n-1)}$ linearly interpolated on a finer grid, thus

$$
R=\sum_{i, j=1}^{N_{\mathrm{x}}, N_{\mathrm{y}}}\left(\kappa_{i j}^{(n)}-\kappa_{i j}^{(n-1)}\right)^{2} .
$$

For the case of $n=0$ we use an initial guess for $\kappa$ which can in practice be obtained from strong lensing, direct finite-field reconstruction, parametrised model fitting to weak lensing data, or simply set to a constant. This form of regularisation is relatively easy to implement and efficient in penalising the small-scale fluctuations in $\kappa$. If enough iteration 
steps are used it does not affect the mass-sheet degeneracy, since the information on the initial $\kappa^{(0)}$ is lost and the initial guess can not bias the results.

\section{Testing the reconstruction method using simulations}

To generate the mock catalogues we use a cluster from the high-resolution N-body simulation by Springel et al. (2001). The cluster we use is taken from the S4 simulation (for details see the aforementioned paper). We constructed the mock catalogues that closely follow the quality of the data (i.e. number density, redshift distribution, errors on ellipticity measurements) we use in the next section. The two different type of catalogues thus have the spatial and redshift distribution equal to the I and R-band catalogues of RX J1347-1145. We generate a quadruply imaged system at a redshift of $z_{\mathrm{s}}=1.76$ and use it in the strong lensing constraints for the reconstruction. The image positions and the original $\kappa$-map (for the cluster at a redshift of $z_{\mathrm{d}}=0.4$ ) are given in Fig. 1a, the reconstructed $\kappa$ is given in Fig. $1 b$.

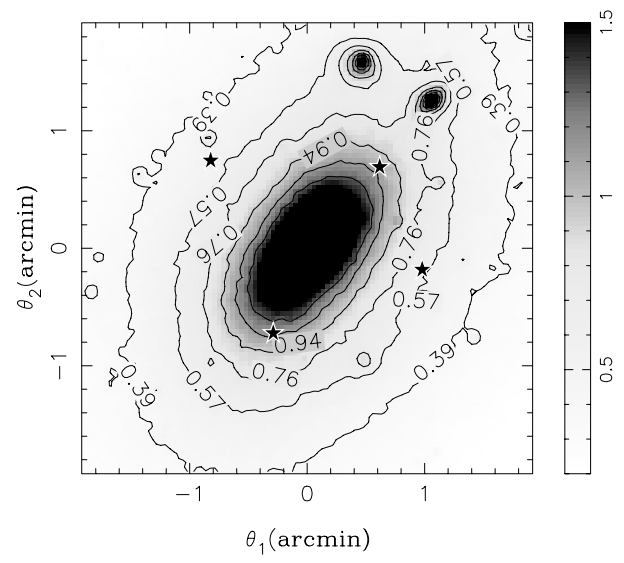

(a)

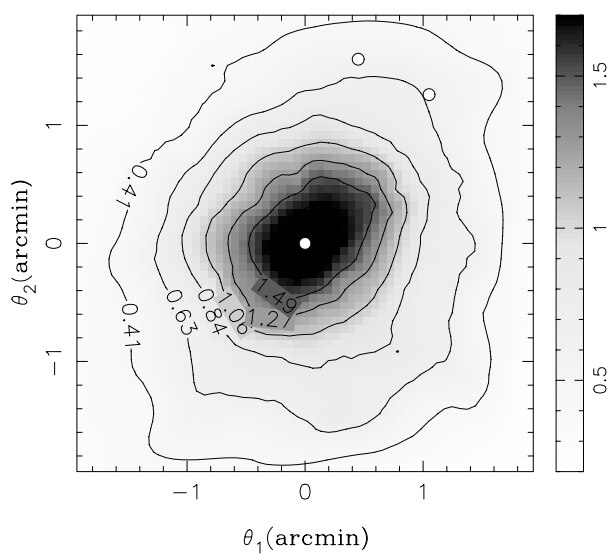

(b)

Figure 1. a) The surface mass density $\kappa$ (for a fiducial source at $z_{\mathrm{s}} \rightarrow \infty$ of the simulated cluster used to generate mock catalogues. The stars in a) denote the image positions of a four-image system at $z_{\mathrm{s}}=1.76$ we use for the reconstruction. b) The reconstructed $\kappa$-map (for a fiducial source at $z_{\mathrm{s}} \rightarrow \infty$ ) from $N_{\mathrm{g}}=210$ galaxies following the properties of the weak lensing catalogue used in Sect. 4. Here we use the initial conditions on $\kappa^{(0)}$ from strong lensing.

From the reconstructed maps we have also estimated the mass within the radius of $1^{\prime} .5\left(340 h^{-1} \mathrm{kpc}\right)$ around the cluster centre. For this purpose we generated 10 mock catalogues for each band and did the reconstruction again with three different initial conditions. All the mass estimates are similar; note, however, that the two galaxy catalogues have galaxy positions and redshifts partly in common and the errors are therefore correlated. We find the enclosed mass of the simulated cluster to be $(1.0 \pm 0.1) \times 10^{15} M_{\odot}$, which is very close to the input value of $M_{\mathrm{s}}\left(<340 h^{-1} \mathrm{kpc}\right)=0.99 \times 10^{15} M_{\odot}$. The error has been estimated from the variance of mass determinations form different mock catalogues. The results show that our method is effectively able to break the mass-sheet degeneracy and is, as a consequence, very efficient in reproducing the cluster mass also at radii different from the Einstein radius of the cluster. Note that a single multipleimage system does not by itself break this degeneracy, since the transformation does not affect the image positions (and the same is true for the magnification ratios). We would need at least two different redshift multiple image systems to break the mass-sheet 
degeneracy with strong lensing data alone. It is also very encouraging that the results are independent of the initial $\kappa^{(0)}$ used for the regularisation.

Unfortunately we can not resolve both clumps present in the simulations. This is due to the fact that the number density of background sources is low and the internal smoothing scale (i.e. the average distance between two source galaxies) is large; with a number density of $\sim 100 / \operatorname{arcmin}^{2}$ the clumps can be easily resolved.

\section{Cluster mass reconstruction of RX J1347-1145}

In this section we present the mass modelling of the most X-ray-luminous cluster RX J1347-1145 (Schindler et al. 1995). Due to its record holding this cluster has been a subject of many studies in X-rays, optical as well as at radio ( $\mathrm{mm}$ ) frequencies. Unfortunately studies based on X-ray properties, SZ-effect, velocity dispersion measures, strong and weak lensing have yielded discrepant results for the mass estimate (see e.g. Cohen \& Kneib 2002 for a summary).

Encouraged by the success of the tests of our cluster mass reconstruction method, we apply it to VLT/FORS data on the field of $3.8 \times 3.8 \mathrm{arcmin}^{2}$ in UBVRI bands. These five colours were also used for the photometric redshift estimates of the source galaxies in weak lensing. In addition, we use J and H-band data from SOFI and K-band data from ISAAC to improve the photometric redshift estimates for the strong lensing candidates (in the inner regime of the cluster).

\subsection{Multiple image systems of $R X J 134{ }^{7-1145}$}

Thus far, five arcs have been reported in the cluster. The first two were discovered by Schindler et al. (1995), and HST images revealed three additional arcs (Sahu et al. 1998). Unfortunately, these five arcs (A1-A5 as labelled by Sahu et al. 1998) do not belong to the same multiple image system. However two of them (A4 and A5) do have the same colours and we consider them to belong to the same multiply imaged system. Based on the colours and surface brightness of the images in the field we find a possible candidate counter image which we include in the analysis. Using 8 colours for the redshift determination of A4-A5 and 5 colours for the counter image (it is located at the edge of the $\mathrm{J}, \mathrm{H}$, and $\mathrm{K}$-band images and therefore the photometry is not reliable) we find all three images are consistent with being at a redshift of $z_{\mathrm{s}} \simeq 1.76$.

To obtain $\kappa^{(0)}$ we fit a non-singular isothermal ellipse model to the strong lensing data. In addition to the image positions, we also use image ellipticities as constraints. We allow the centre of the potential $\vec{\theta}_{\mathrm{cl}}$, the scaling $b_{0}$, ellipticity $\left|\epsilon_{\mathrm{g}}\right|$, and position angle $r_{\mathrm{c}, \text { nis }}$ to vary. Following the prescription of Kneib et al. (1996) we also include the ten brightest cluster members (selected according to their I-band magnitude and having photometric redshifts estimate between 0.4 and 0.5 ) to the model. They have been modelled as nonsingular isothermal spheres with line-of-sight velocity dispersion $\sigma_{\text {nis }}$ and core radius $r_{\mathrm{c}, \text { nis }}$ following $\sigma_{\text {nis }} \propto L^{1 / 4}$ and $r_{\mathrm{c}, \text { nis }} \propto L^{1 / 2}$. We stress, however, that the only aim of this modelling was to obtain the initial model for $\kappa^{(0)}$. Therefore we also do not include additional strong lensing candidates; for them one would need good redshift information. The multiple-image system described here will be further included in the combined strong and weak lensing reconstruction described in the next section.

\subsection{Combined weak and strong lensing mass reconstruction of $R X J 1347-1145$}

Finally we apply our mass-reconstruction method to the I and R-band weak lensing data of a redshift $z_{\mathrm{d}}=0.451$ cluster RX J1347-1145 consisting of $N_{\mathrm{g}}=210$ and $N_{\mathrm{g}}=$ 145 galaxies respectively. In addition we use the multiply imaged system described in 
Sect. 4.1. We use three different initial models for $\kappa^{(0)} ; I M$ comes from the best-fit model from the strong lensing analysis of the cluster, the $I S$ model is the best-fit SIS model to binned tangential ellipticities (centred on the brightest cluster member) and $I 0$ has $\kappa^{(0)}=0$. The regularisation parameter was set to $\eta=400(200)$ in all three cases for I and R-band respectively.

The resulting $\kappa$-maps for the $I M$ initial model for I and $\mathrm{R}$ band weak lensing data are given in Fig. 2. We determine the enclosed mass within a radius of $1^{\prime} .5$ (for $z_{\mathrm{d}}=0.45$ corresponding to $360 h^{-1} \mathrm{kpc}$ ) for both bands and all three initial model as follows:

$$
\begin{array}{lll}
I M: & M_{I}=1.46 \times 10^{15} M_{\odot} \text { and } & M_{R}=1.36 \times 10^{15} M_{\odot} \\
I S: & M_{I}=1.19 \times 10^{15} M_{\odot} \text { and } & M_{R}=1.19 \times 10^{15} M_{\odot} \\
I 0: & M_{I}=1.10 \times 10^{15} M_{\odot} \text { and } & M_{R}=1.03 \times 10^{15} M_{\odot}
\end{array}
$$

We estimate the mass of the cluster to be $M\left(<360 h^{-1} \mathrm{kpc}\right)=(1.2 \pm 0.3) \times 10^{15} M_{\odot}$. The error and the differences between reconstructions with different initial models are larger than in the case of simulated images. This is mostly attributed to the fact that we only use a three-image system. In addition, when computing the redshifts for the mock catalogues we did not include the outliers (i.e. galaxies with redshift estimates which can be wrong by more than factor 2). However, within the given error the results for both bands and for different initial models are consistent.

The resulting mass is larger than obtained by velocity dispersion measurements of Cohen \& Kneib (2002). It is, however, consistent with X-ray data by Allen et al. (2002) and the previous weak-lensing results by Fischer \& Tyson (1997). We note however, that our results depend upon the correct redshift determination and identification of the members of the multiple image system we use. If we put the multiple image system to a redshift of $\sim 3(\sim 1.3)$ the estimated mass decreases(increases) by $\sim 10 \%$. If the images do not belong to the same system, the changes might be even more drastic. However, at least for the two arcs A4 and A5 we consider this possibility unlikely.
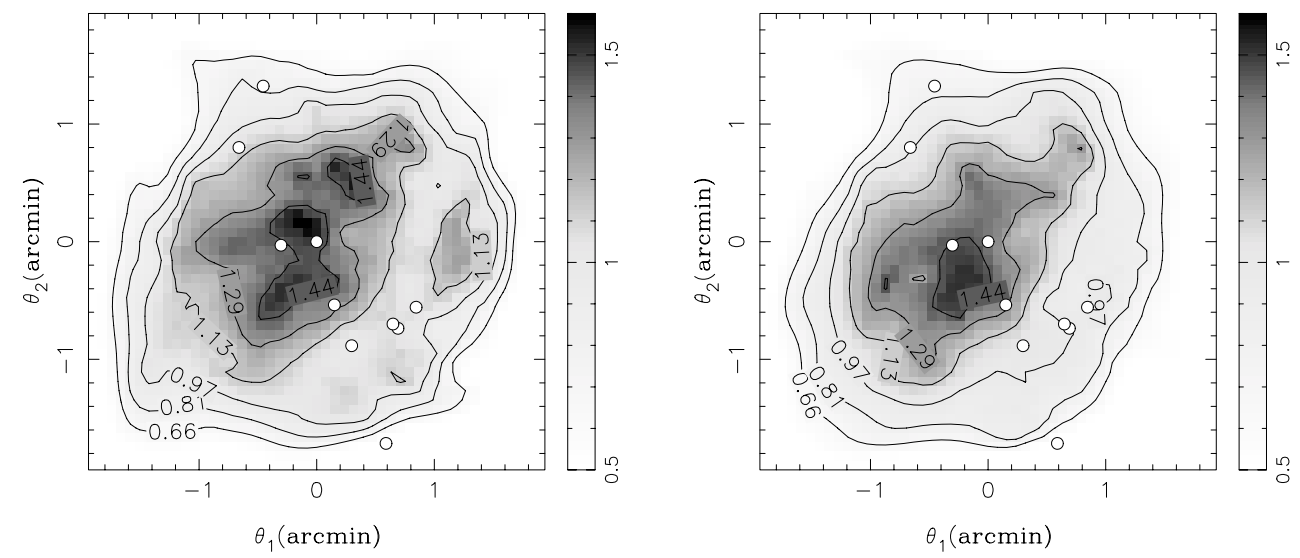

Figure 2. Left I-band and right R-band reconstructed $\kappa$-maps obtained using weak and strong lensing data of the cluster RX J1347-1145 with known photometric redshifts. We use the best-fit model from the strong lensing analysis of the cluster for $\kappa^{(0)}$. The regularisation parameter was set to $\eta=400$ for I and $\eta=200$ for the R-band data. The positions of 10 brightest cluster members (in I-band) are plotted as white circles.

\section{Conclusions}

We have developed a potential reconstruction method to determine the cluster mass and mass-distribution that uses the combined constraints from weak and strong lensing. 
We test the method on simulated data and conclude that it is very successfully in reconstructing the cluster mass and mass distribution. We determine the enclosed mass within $340 h^{-1} \mathrm{kpc}$ of the simulated cluster to be $(1.0 \pm 0.1) \times 10^{15} M_{\odot}$, which is very close to the input value of $M_{\mathrm{s}}\left(<340 h^{-1} \mathrm{kpc}\right)=0.99 \times 10^{15} M_{\odot}$. With the data quality we use we are therefore effectively able to break the mass-sheet degeneracy and therefore obtain the mass and mass-distribution estimates without prior assumptions on the lensing potential.

In the second part we apply the method to the weak and strong lensing data of RX J1347-1145 and reach the following conclusions.

(a) The combined reconstruction confirms that the most X-ray luminous cluster is indeed very massive. If the redshift and identification of the multiple-image system is correct we estimate the enclosed cluster mass within $360 \mathrm{~h}^{-1} \mathrm{kpc}$ to $M\left(<360 \mathrm{~h}^{-1} \mathrm{kpc}\right)=$ $(1.2 \pm 0.3) \times 10^{15} M_{\odot}$.

(b) The reconstruction shows a south-east mass extension, comparable to the X-ray measurements and a possible north-west one.

The mass-reconstruction of RX J1347-1145 can be significantly improved. Deep HST imaging would greatly help in identifying and confirming new multiple-image systems that we can use, thus allowing even more detailed modelling. In addition, not only the centre of the light for each of the arcs can be used as constraints, but also their morphology. The reconstruction technique with an adaptive grid at the image positions can be used for these purposes and will be a subject of future work. Further, spectroscopic redshifts need to be obtained for the multiple-image system candidates. Deep, wide-field imaging data of this cluster will help us improve the weak lensing constraints and the reconstruction can be performed at larger radii than presented here.

The method has thus shown a high potential for the future. If highest-quality data is used (higher number density of background sources and additional multiply imaged systems), a combination of strong and weak lensing has proven to offer a unique tool to pin down the masses of galaxy-clusters as well as their profiles and accurately test predictions within the CDM framework.

\section{Acknowledgements}

We would like to thank Léon Koopmans, Oliver Czoske, and Douglas Clowe for many useful discussions and Volker Springel for providing us the N-body simulations. This work was supported by the graduate schools IMPRS at MPIfR Bonn and BIGS at University of Bonn.

\section{References}

Allen, S. W., Schmidt, R. W., \& Fabian, A. C. 2002, MNRAS, 335, 256

Bartelmann, M., Narayan, R., Seitz, S., \& Schneider, P. 1996, ApJ, 464, L115

Bradač, M., Schneider, P., Erben, T., \& Lombardi. 2004a, in prep.

Bradač, M., Schneider, P., Erben, T., et al. 2004b, in prep.

Cohen, J. G. \& Kneib, J. 2002, ApJ, 573, 524

Fischer, P. \& Tyson, J. A. 1997, AJ, 114, 14

Kneib, J.-P., Ellis, R. S., Smail, I., Couch, W. J., \& Sharples, R. M. 1996, ApJ, 471, 643

Sahu, K. C., Shaw, R. A., Kaiser, M. E., et al. 1998, ApJ, 492, L125

Schindler, S., Guzzo, L., Ebeling, H., et al. 1995, A\&A, 299, L9

Seitz, S., Schneider, P., \& Bartelmann, M. 1998, A\&A, 337, 325

Springel, V., White, S. D. M., Tormen, G., \& Kauffmann, G. 2001, MNRAS, 328, 726 